K.G. Binmore and R. Hornblower

Nagoya Math. J.

Vol. 48 (1972), 173-181

\title{
BOUNDARY BEHAVIOUR OF FUNCTIONS WITH HADAMARD GAPS
}

\author{
K. G. BINMORE AND R. HORNBLOWER
}

1. Introduction. In this paper we discuss the boundary properties of a function $f$ which is analytic in the open unit disc $\Delta$ and has Hadamard gaps-i.e.

$$
f(z)=\sum_{n=0}^{\infty} a_{n} z^{\lambda_{n}} \quad(z \in \Delta)
$$

where

$$
\frac{\lambda_{n+1}}{\lambda_{n}} \geq q>1 \quad(n=1,2,3, \cdots) .
$$

This gap structure inhibits the possibility of cancellation in the Taylor series. Indeed, M. Weiss [11] has shown that, under appropriate growth conditions on the coefficients, the partial sums of the series (1) behave like independent random variables on $\partial \Delta$ and, in particular, that a law of the iterated logarithm holds.

The behaviour of $f$ must therefore be expected to be highly irregular all around the boundary of the unit disc. In this sense, the boundary properties of functions with Hadamard gaps are 'typical' of the boundary properties of 'almost all' functions analytic in $\Delta$. (See Offord [9].)

Suppose firstly that $\sum\left|a_{n}\right|<\infty$. Then $f$ is continuous on $\bar{\Delta}$ and there is nothing more to be said. We exclude this trivial case and assume from now on that

$$
\sum\left|a_{n}\right|=\infty
$$

Under this hypothesis, $\partial \Delta$ is a natural boundary for $f$ (Ostrowski [10]). One may ask, what is the range of $f$ ? Presumably this is the whole of the complex plane covered infinitely often. However, in the

Received June 26, 1971. 
general case, this conjecture remains unproven. It is known to be true in the case when $q$ is large (M. and G. Weiss [13]) and also for arbitrary $q>1$, provided that $a_{n} \nrightarrow 00(n \rightarrow \infty)$ (W. Fuchs [5]).

One may also ask about the asymptotic properties of $f$. Here there is a sharp division into two cases.

Under the hypothesis that $a_{n} \rightarrow 0(n \rightarrow \infty)$, we have that, given any $\omega \neq \infty$, the set of points $\zeta \in \partial \Delta$ such that

$$
\sum a_{n} \zeta^{\lambda_{n}}=\omega
$$

is uncountable and dense on $\partial \Delta$. This is a theorem of Paley and M. Weiss [12]. The set of $\zeta$ for which (4) is true must in fact be rather thin since, in view of Collingwood's maximality theorem [4, p. 80], it is of first category in $\partial \Delta$. (See the result of Stechkin quoted in Bary, [2, p. 25]).

Alternatively, we have the case $a_{n} \neq 0$ as $n \rightarrow \infty$. In this case $f$ can have no finite asymptotic values at all (Binmore [3]). One assumes that if $\omega$ is an asymptotic value of $f$ at $\zeta$, then (4) holds. But this is not known in general.

There remains the question of the asymptotic value $\infty$. It follows from the theorem of Paley and Weiss that a bounded $f$ must have $\sum\left|a_{n}\right|<\infty$. It is therefore certainly true that $\infty$ is an asymptotic value when $\sum\left|a_{n}\right|=\infty$. A question which has been raised by Anderson [1], among others, is the following. Is it true that $f$ has $\infty$ as an asymptotic value at a set of points which is dense on $\partial \Delta$ ?

In the case when $a_{n} \not>0(n \rightarrow \infty)$, this is the the same question as asking whether or not $f$ belongs to Maclane's class $\mathscr{A}$. This is the class of non-constant functions which are analytic in $\Delta$ and have asymptotic values at a dense set of points on $\partial \Delta$. It is known that a non-constant function analytic in $\Delta$ belongs to $\mathscr{A}$ provided that

$$
\int_{0}^{1} \log ^{+} \log ^{+} M(r) d r<\infty
$$

where $M(r)$ denotes the maximum modulus of the function on the circle $|z|=r . \quad$ (Hornblower [6]).

As an example of a function of unrestricted growth which belongs to $\mathscr{A}$, we have the function $f$ given by (1) with $q>3$ (Maclane [8, p. 46]). Maclane's proof extends to the cases $q=2$ and $q=3$ but the case of 
arbitrary $q>1$ seems to be more difficult. In this paper, we offer a partial solution to this question.

Let $\nu(r)$ denote the central index of the function $f$-i.e. the largest exponent $\lambda_{m}$ with the property that

$$
\left|a_{m} r^{\lambda_{m}}\right|=\max _{n}\left|a_{n} r^{\lambda_{n}}\right|
$$

The central index is an increasing function of $r$ and is continuous on the right for $r<1$. We then have

THEOREM 1. Suppose that $f$ is given by (1) and (2) and that

$$
\frac{\dot{\nu}(r)}{\nu\left(r^{-}\right)} \leq Q<\infty \quad(0<r<1) .
$$

Then $f$ belongs to Maclane's class $\mathscr{A}$.

In contrast with the result of Maclane described above, which asserts that $f \in \mathscr{A}$ as soon as the gaps are sufficiently large, this result says, roughly speaking, that $f \in \mathscr{A}$ when the gaps are not too big. For example, if the non-zero coefficients of $f$ increase in modulus and

$$
1<q \leq \frac{\lambda_{n+1}}{\lambda_{n}} \leq Q<\infty \quad(n=1,2, \cdots)
$$

then it follows from Theorem 1 that $f \in \mathscr{A}$.

In spite of the difficulty of proving the result even in the case of Hadamard gaps, one might expect to obtain the conclusion $f \in \mathscr{A}$ from a much weaker gap hypothesis than (2). We suggest that the condition

$$
\sum \lambda_{n}^{-1}<\infty
$$

may be sufficient and, probably, even this is too strong. There is some reason to suppose further that, under the same hypothesis (7), there is a non-trivial $\left\{\varepsilon_{n}\right\}$, possibly

$$
\varepsilon_{n}=\prod_{\substack{k=0 \\ k \neq n}}^{\infty}\left|\frac{\lambda_{n}-\lambda_{k}}{\lambda_{n}+\lambda_{k}}\right|,
$$

with the property that $f$ can have no finite asymptotic value unless $\varepsilon_{n} a_{n} \rightarrow 0(n \rightarrow \infty)$. (See Binmore [3]).

If these suggestions are true we can deduce the following result, which we quote below as a conjecture, by means of a method of Anderson [1]. 
CONJECTURE. Suppose that $\sum \lambda_{n}^{-1}<\infty$. Then there is a non-trivial sequence $\left\{\varepsilon_{n}\right\}$ with the property that if $f$, given by (1), satisfies

$$
\varepsilon_{n} a_{n} \nrightarrow 0 \quad(n \rightarrow \infty)
$$

then the closure of the set of zeros of $f$ contains the whole of $\partial \Delta$.

We consider the zeros of $f$ rather than any other value simply for convenience. Fuchs has recently shown this conjecture to be true with $\varepsilon_{n}=1(n=1,2, \cdots)$ in the case of Hadamard gaps. This approach is entirely different from that suggested above. He suggests that the hypothesis $a_{n} \nrightarrow 0(n \rightarrow \infty)$ of his result should be replaced by $\sum\left|a_{n}\right|=\infty$.

It is at least clear that, under the sole hypothesis $\sum\left|a_{n}\right|=\infty$, the cluster set $C\left(f, e^{i \theta}\right)$ is total for each $\theta$. (The case when $a_{n} \rightarrow 0$ is contained in the theorem of Paley and Weiss). It follows, incidentally, from Collingwood's maximality theorem that, if $\sum\left|a_{n}\right|=\infty$, then the set of points at which $f$ has a radial asymptotic value (finite or infinite) is of first category in $\partial \Delta$.

\section{Proof of Theorem 1}

We shall use the letter $K$ to denote a set which consists of the union of a set of continua lying in $\Delta$ such that each continuum has diameter exceeding some fixed positive number $\delta$ and, further, such that $K$ has at least one limit point on $\partial \Delta$. In particular, $K$ may be a Koebe sequence of arcs (see, for example, Maclane [8]).

We deduce Theorem 1 from

THEOREM 2. Suppose that (6) holds. Then

$$
\left|a_{n}\right| \leq M \sup _{z \in K}|f(z)| \quad(n=1,2, \cdots)
$$

for some constant $M<\infty$.

We now show how to deduce Theorem 1 from Theorem 2. Recall that the level sets $L(\lambda)$ of $f$ are defined, for each $\lambda>0$, by

$$
L(\lambda)=\{z \in \Delta|| f(z) \mid=\lambda\} .
$$

Let $S$ be a subset of $\Delta$ and let $\delta(r)(0<r<1)$ denote the supremum of the diameters of the components of the set $S \cap\{\mathrm{r}<|z|<1\}$. If $\delta(r) \rightarrow 0(r \rightarrow 1-)$, then $S$ is said to 'end at points' on $\partial \Delta$. Maclane [8] has shown that the class $\mathscr{A}$ is identical with the class of those non- 
constant functions which are analytic in $\Delta$ and whose level sets end at points on $\partial \Delta$.

Now suppose that the conclusion of Theorem 2 holds. If $f \notin \mathscr{A}$, then, for some $\lambda>0$, the level set $L(\lambda)$ does not end at points on $\partial \Delta$ and therefore contains a subset $K$ satisfying the hypotheses of Theorem 2. Thus

$$
\left|a_{n}\right| \leq M \sup _{z \in L(\lambda)}|f(z)|=M \lambda \quad(n=1,2,3, \cdots) .
$$

This implies that $f$ satisfies (5) and hence that $f \in \mathscr{A}$. This is a contradiction, and hence Theorem 2 implies Theorem 1 .

\section{Proof of Theorem 2 .}

The arguments used to establish Theorem 2 are similar to those in [3]. Specifically, we require the following lemma.

LEMMA 1. Let $P$ be a given positive integer and let $\nu_{0}, \nu_{1}, \cdots \nu_{P}$ be any complex numbers satisfying

(i) $\operatorname{Re} \nu_{k} \geq \operatorname{Re} \nu_{0}=P$

(ii) $\left.\left|\nu_{k}-\nu_{0}\right| \leq k / P \quad\right\}(j, k=0,1, \ldots P)$

(iii) $\left|\nu_{k}-\nu^{j}\right| \geq|k-j| / P \mid$

Then there exists an exponential polynomial

$$
G_{P}(x)=\sum_{k=0}^{P} g_{k} e^{-\nu_{k} x}
$$

satisfying

$$
\begin{aligned}
& \text { (i) }\left|G_{P}(x)\right| \leq 2\left\{x e^{1-x}\right\}^{P} \quad(x \geq 0) \\
& \text { (ii) } G_{P}(1)=1 \\
& \text { (iii) } \sum_{k=0}^{P}\left|g_{k}\right| \leq 2(2 e P)^{P}
\end{aligned}
$$

We now embark on the proof of Theorem 2. In accordance with the notation of [3], we begin with the transformation $z=e^{-\omega}$. The set $K$ is then transformed into a set $J$ consisting of the union of a set of continua lying in the right half plane. Each of these continua has diameter exceeding some fixed positive number $\delta$ and their union $J$ has a limit point on the imaginary axis.

Now consider the values of the function 


$$
F(\omega)=\sum_{n=0}^{\infty} a_{n} e^{-\lambda_{n} \omega}
$$

on the set $J$. Note, in particular, that

$$
\sup _{z \in K}|f(z)|=\sup _{\omega \in J}|F(\omega)| \text {. }
$$

We denote the central index of $f$ as a function of $x=\operatorname{Re} \omega$ by $N$. Thus $N(x)=\nu\left(e^{-x}\right)$. The function $N(x)$ is decreasing and continuous on the left and hypothesis (6) is equivalent to

$$
\frac{N(x)}{N(x+)} \leq Q<\infty \text {. }
$$

We may assume that $N(x) \rightarrow+\infty(x \rightarrow 0+)$ for otherwise it follows immediately that the coefficients are bounded.

Let $X$ be any positive number for which

$$
\frac{1}{N(x)}<\frac{1}{2} \delta \quad(0<x<X)
$$

and let $\alpha$ be a component of $J$ which contains points to the left of the line $\operatorname{Re} \omega=X$. Such a component exists since $J$ has a limit point on the imaginary axis. Let $\zeta=\xi+i \eta$ be a point of smallest real part on $\alpha$. Then it is clear that there exists a point $\zeta^{\prime}$ on $\alpha$ whose distance from $\zeta$ is at least $\frac{1}{2} \delta$.

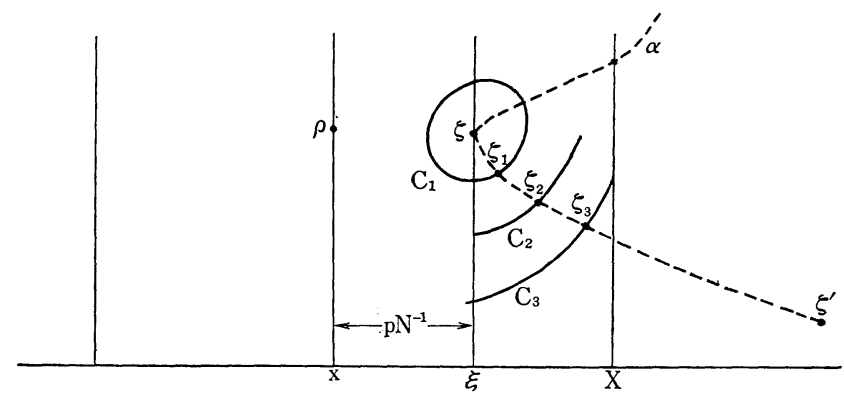

Figure 1

Now let $p$ be a positive integer (later to be chosen suitably). Then the function

$$
g(x)=x+\frac{p}{N(x)}
$$


is monotonic increasing and continuous on the left. Since $g(\xi)>\xi$ and $g(x) \rightarrow 0(x \rightarrow 0+)$ there exists an $x(0<x<\xi)$ such that

$$
g(x) \leq \xi \leq g(x+) \text {. }
$$

We choose $P$ so that

$$
x+P / N(x)=\xi .
$$

It follows from (12) and hypothesis (10) that

$$
p \leq P \leq p Q \text {. }
$$

Now let $\rho=x+i \eta$. (Recall that $\zeta=\xi+i \eta$ ). Write $A_{n}=a_{n} e^{-\rho \lambda_{n}}$ $(n=1,2,3, \cdots)$. Then, if $N(x)=\lambda_{m}$,

$$
\left|A_{m}\right|=\max _{n}\left|A_{n}\right|
$$

Also, we have that

$$
F_{\rho}(\omega)=F(\omega+\rho)=\sum_{n=0}^{\infty} A_{n} e^{-\lambda_{n} \omega} .
$$

It follows that, if $\nu_{0}, \nu_{1}, \cdots \nu_{P}$ are complex numbers satisfying (8),

$$
\begin{aligned}
T(x) & =\sum_{k=0}^{P} g_{k} F_{\rho}\left(\nu_{k} x\right)=\sum_{k=0}^{P} g_{k} \sum_{n=0}^{\infty} A_{n} e^{-\lambda_{n} \nu_{k} x} \\
& =\sum_{n=0}^{\infty} A_{n} \sum_{k=0}^{P} g_{k} e^{-\lambda_{n} \nu_{k} x}=\sum_{n=0}^{\infty} A_{n} G_{P}\left(\lambda_{n} x\right) .
\end{aligned}
$$

Consequently

$$
\begin{aligned}
& \left|T\left(\frac{1}{\lambda_{m}}\right)\right|\left|\sum_{n=0}^{\infty} A_{n} G_{P}\left(\frac{\lambda_{n}}{\lambda_{m}}\right)\right| \\
& \quad \geq\left|A_{m}\right|\left\{1-\sum_{n \neq m}\left|G_{P}\left(\frac{\lambda_{n}}{\lambda_{m}}\right)\right|\right\} \\
& \quad \geq\left|A_{m}\right|\left\{1-2 \sum_{s=1}^{\infty}\left(q^{-s} e^{1-q^{-s}}\right)^{p}-2 \sum_{s=1}^{\infty}\left(q^{s} e^{1-q^{s}}\right)^{p}\right\}
\end{aligned}
$$

Since, by (9i) and (14), $G_{P}(x) \leq\left(x e^{1-x}\right)^{p} \leq\left(x e^{1-x}\right)^{p}$. It follows that, if $p$ is chosen sufficiently large, then

$$
\frac{1}{2}\left|A_{m}\right| \leq\left|T\left(\frac{1}{\lambda_{m}}\right)\right| \text {. }
$$

Note that the choice of $p$ depends only on the value of $q$.

We now choose the numbers $\nu_{0}, \nu_{1}, \cdots \nu_{P}$. Define $\zeta_{0}=\zeta$ and draw 
circles $C_{k}(k=1,2, \cdots P)$ with common centre $\zeta_{0}$ and radii $k\left(P \lambda_{m}\right)^{-1}$ respectively. It is clear that there exist points $\zeta_{1} \cdots \zeta_{P}$ such that

$$
\left.\begin{array}{l}
\text { (i) } \zeta_{k} \in \alpha \\
\text { (ii) } \zeta_{k} \in C_{k} \\
\text { (iii) } R \zeta_{k} \geq R \zeta_{0}
\end{array}\right\}(k=1, \cdots, P)
$$

(see figure 1). Note that (11) ensures that

$$
\left|\zeta_{0}-\zeta_{P}\right|=\frac{1}{N(x)}<\frac{1}{2} \delta \leq\left|\zeta-\zeta^{\prime}\right|
$$

Now set

$$
\nu_{k}=\lambda_{m}\left(\zeta_{k}-\rho\right) \quad(k=0,1, \ldots P) .
$$

Then conditions (8) of Lemma 1 are satisfied and so we may deduce from (16), (17) and (9iii) that

$$
\begin{aligned}
\frac{1}{2}\left|A_{m}\right| & \leq\left|T\left(\frac{1}{\lambda_{m}}\right)\right| \leq \sum_{k=0}^{P}\left|g_{k}\right| \cdot\left|F_{\rho}\left(\frac{\nu_{k}}{\lambda_{m}}\right)\right|=\sum_{k=0}^{P}\left|g_{k}\right| \cdot\left|F\left(\zeta_{k}\right)\right| \\
& \leq 2(2 e P)^{P} \sup _{\omega \in \alpha}|F(\omega)| \leq 2(2 e P)^{P} \sup _{\omega \in J}|F(\omega)| .
\end{aligned}
$$

From (15) it follows that, for $n=1,2, \cdots$,

$$
\left|A_{n}\right|=\left|a_{n} e^{-\lambda_{n} x}\right| \leq M \sup _{\omega \in J}|F(\omega)|
$$

where the constant $M$ may be taken by (14) to be $4(2 e p Q)^{p Q}$ and hence depends only on the values of $q$ and $Q$.

The inequality (18) holds for some value of $x$ satisfying $0<x<X$. The positive constant $X$ is restricted only by (11) which will be satisfied for all small enough values of $X$. We may therefore allow $x$ to tend to zero through a suitable sequence in (18) and obtain

$$
\left|a_{n}\right| \leq M \sup _{\omega \in J}|F(\omega)|=M \sup _{z \in K}|f(z)|
$$

This completes the proof of Theorem 2 .

\section{REFERENCES}

[1] J. M. Anderson, 'Boundary properties of analytic functions with gap power series', Quart. J. Math. (2) (Oxford), 21 (1970), 247-256.

[2] N. Bari, 'A Treatise on Trigonometric Series, Vol. II', New York (1964).

[ 3 ] K. G. Binmore, 'Analytic functions with Hadamard gaps', Bull. London Math. Soc. 1 (1969), 211-217. 
[4] E. F. Collingwood and A. J. Lohwater, 'The Theory of Cluster Sets', C.U.P. (1966).

[ 5 ] W. H. J. Fuchs, 'On the zeros of a power series with Hadamard gaps', Nagoya Math. J., 29 (1967), 167-174.

[ 6 ] R. Hornblower, 'A growth condition for the MacLane class $\mathscr{A}$ ', Proc. Lond. Math. Soc. (3) 23 (1971), 371-384.

[ 7 ] J.-P. Kahane and M. and G. Weiss, 'On lacunary power series', Ark. Math., 5 (1963), 1-26.

[ 8 ] G. R. Maclane, 'Asymptotic Values of Holomorphic Functions', Rice University Studies, Vol. 49, No. 1, (1963).

[9] A. C. Offord, 'The distribution of the zeros of power series whose coefficients are independent random variables', Indian J. Math., 9 (1967), 175-196.

[10] A. Ostrowski, 'Uber eine Eigenschaft gewisser Potenzreihen mit unendlich vielen verschiedenen Koeffizienten', Berlin Ber. 34 (1921), 557-565.

[11] M. Weiss, 'The law of the iterated logarithm for lacunary trigonometric series', Trans. Amer. Math. Soc., 91 (1959), 444-469.

[12] M. Weiss, 'Concerning a theorem of Paley on lacunary power series', Acta Math. 102 (1959), 225-238.

[13] M. and G. Weiss, 'On the Picard property of lacunary power series', Studia Math. (1962-63), 221-245.

London School of Economics,

Math. Dept.

S.U.N.Y.A., 